\title{
Quadratic Inference Functions for Varying Coefficient Models with Longitudinal Data
}

\author{
Annie $Q u^{*}$ and \\ Department of Statistics, Oregon State University, Corvallis, Oregon 97331 \\ Runze Li \\ Department of Statistics, Pennsylvania State University, University Park, PA 16802
}

\section{Summary}

Nonparametric smoothing methods are used to model longitudinal data, but the challenge remains to incorporate correlation into nonparametric estimation procedures. In this paper, we propose an efficient estimation procedure for varying coefficient models for longitudinal data. The proposed procedure can easily take into account correlation within subjects and directly deal with both continuous and discrete response longitudinal data under the framework of generalized linear models. Unlike the generalized estimation equation approach, the newly proposed procedure is more efficient when the working correlation is misspecified. For varying-coefficient models, it is often of interest to test whether coefficient functions are time-varying or time-invariant. We propose a unified and efficient nonparametric hypothesis testing procedure, and further demonstrate that the resulting test statistics have an asymptotic chi-squared distribution. In addition, the goodness-of-fit test is applied to test whether the model assumption is satisfied. The corresponding test is also useful for choosing basis functions and the number of knots for regression spline models in conjunction with the model selection criterion. We evaluate the finite sample performance of the proposed procedures with Monte Carlo simulation studies. The proposed methodology is illustrated by an analysis of a AIDS data set.

\section{Keywords}

Generalized method of moments; Goodness-of-fit; Model selection; Penalized spline; Quadratic inference function; Smoothing spline; Varying-coefficient model

\section{Introduction}

Longitudinal data occur often in biomedical research, where data are collected at irregular and possibly subject-specific time points. Due to their unbalanced nature, it is difficult to directly apply traditional multivariate regression techniques. To explore possible time-dependent effects, time-varying coefficient models and their extensions have been proposed for longitudinal data analysis. See, for example, Hoover, et al. (1998), Wu, et al. (1998), Fan and Zhang (2000), Martinussen and Scheike (2001), Chiang, et al. (2001), Huang, et al. (2002) and references therein. These authors propose various estimation procedures for varyingcoefficient models under the settings of longitudinal data, but they have not discussed how to incorporate information on the correlation structure within subjects into their estimation procedures. Furthermore, the aforementioned works only discuss continuous responses under a linear model framework. In this paper, we are interested in developing a general approach for both continuous and discrete responses under the generalized linear model framework. 
There are vast nonparametric approaches for correlated errors (Wang, 1998a and 1998b; Opsomer et al., 2001 and references therein), but most of nonparametric literature focus on consistent and efficient estimation including recent series of kernel and spline approaches by Lin and Carroll (2000), Wang (2003), Lin et al. (2004) and Wang et al. (2005). Hart (1997) and references therein provides nonparametric goodness-of-fit tests, however, most of these approaches treated response variables as normal outcomes. More recently, Zhang (2004) proposed generalized linear mixed models on hypothesis testing for varying coefficients model, the response variables could be non-normal such as binary or poisson, however, there is a strong parametric assumption for random effects, and typically the random effects are assumed to be normal. Since the high dimensional likelihood for correlated discrete data usually does not have a closed form and numerical approximations such as Laplace method might be required. In addition, since Zhang's approach is to transform testing varying coefficient to variance component testing problem, and the variance component has non-negative constraint, therefore Zhang's test is a mixture of chi-squared asymptotically. However, the large sample approximation of mixture chi-squared often performs poor in simulation studies (Crainiceanu and Ruppert, 2004). Testing for more than one variance component could be even more complicated.

Our research is motivated by an analysis of a subset of longitudinal data from the Multi-Center AIDS Cohort study. The data set contains the HIV status of 283 homosexual men who were infected with HIV during the follow-up period between 1984 and 1991. The number and time of measurements vary between individuals, with at least one, to a maximum of 14 , measurements. Huang, et al. (2002) analyzed this data set by taking CD4 cell counts as the response variable, and demonstrated that the effects on baseline intercept, smoking status, age and pre-CD4 counts might be time varying. However, their analysis ignored within subject correlation. It is important to incorporate correlation structures in nonparametric local modeling for longitudinal data, as Wang (2003), Lin et al. (2004) demonstrated that the kernel and smoothing spline estimators using the true covariance is more efficient than the independent structure. However, in practice, the true covariance is often unknown.

Empirically, it is also difficult to estimate an unstructured covariance matrix for the following reasons: (1) the covariance matrix has to be positive definite; however, the estimator of the unstructured covariance matrix is often non-positive definite for unbalanced longitudinal data (Lipsitz, et al. 2000); (2) high dimensional nuisance parameters could be involved if the data are measured over a long period of time; (3) the inverse of the covariance is needed and essential, therefore the smallest eigenvalues of the covariance matrix are the most important; but these are poorly estimated when the dimension of the covariance matrix is large $(\mathrm{Qu}$ and Lindsay, 2003). Even if the correlation matrix is assumed to possess a simplified working structure as in generalized estimating equations (Liang and Zeger, 1986), the estimation of the correlation matrix could still be non-positive definite (Crowder, 1995).

Our goal in this paper is to develop a unified approach which enables us to handle high dimensional problems without losing efficiency. We propose an estimation procedure for varying coefficient models using the penalized spline (Ruppert and Carroll, 2000) and quadratic inference function approaches (Qu, et al. 2000). The proposed method allows us to directly incorporate correlations into model building, but does not require us to estimate the nuisance parameters associated with correlations. Under certain regularity conditions, we establish the asymptotic normality of the resulting estimator and show that our estimator is asymptotically efficient within the class where the moment conditions are satisfied.

Another goal of interest is to examine whether coefficient functions are time-varying or are invariant. In general, this is still challenging as we mention earlier that the likelihood functions are intractable when data are correlated and discrete; in addition, a specific parametric 
alternative is not desirable in nonparametric models, and therefore a typical likelihood ratio test might not be applicable. Huang et al. (2002) constructed test statistics based on the difference of the residual sum of squares under the null and alternative. Their approach does not require likelihood functions; however, the asymptotic properties of their test have not been developed and they proposed bootstrap sampling strategies to determine a critical value.

This leads us to consider nonparametric goodness-of-fit tests for large samples. We propose a simple and efficient statistical inference procedure which does not require likelihood functions. The proposed test statistics have a chi-squared limiting distribution under the null hypothesis. In addition, we are able to perform a goodness-of-fit test for the model assumption. This provides an objective criterion for choosing basis functions in regression spline models and determining the number of knots in penalized spline approaches. A goodness-of-fit test has not been developed in the nonparametric literature when the likelihood function is not available. In addition, we also apply Andrews' (1999) generalized method of moments Bayesian information criterion (BIC) which allows us to select between models when the goodness-offit tests fail to reject.

This paper is organized as follows: In Section 2, we propose an estimation procedure under the varying coefficient model, and further establish the strong consistency and asymptotic normality of the proposed estimator. In Section 3, we discuss some practical issues to implement the proposed estimation procedures. In Section 4, a nonparametric goodness-of-fit test and a model selection procedure are illustrated. We assess the finite sample performance of the proposed procedure with Monte Carlo simulation and illustrate the proposed methodology by an analysis of an AIDS data set in Section 5. Discussion is given in Section 6. Technical conditions and proofs are provided in the Appendix.

\section{A new estimation procedure}

In this section, we will illustrate how to estimate coefficient functions using the penalized spline, and how to incorporate correlation structures using quadratic inference functions. We start with a brief introduction to quadratic inference functions.

\subsection{Quadratic inference functions}

For longitudinal data, let $y_{i}(t)$ be a response variable and $x_{i}(t)$ be a $p \times 1$ vector of covariates, measured at time $t=t_{1}, \ldots, t_{n_{i}}$ for subjects $i=1, \ldots, N$. We assume that the model satisfies the first moment model assumption:

$$
\mu_{i t}=E\left\{y_{i}(t)\right\}=\mu\left\{x_{i}(t)^{\prime} \beta\right\},
$$

where $\mu(\cdot)$ is a known inverse link function and $\beta$ is $p$-dimensional parameter vector. The quasilikelihood equation (Wedderburn, 1974) for longitudinal data is

$$
\sum_{i=1}^{N} \dot{\mu}_{i}^{\prime} V_{i}^{-1}\left(y_{i}-\mu_{i}\right)=0
$$

where $V_{i}=\operatorname{var}\left(y_{i}\right), y_{i}=\left(y_{i}\left(t_{1}\right), \cdots, y_{i}\left(t_{n_{i}}\right)\right)^{\prime}, \mu_{i}=\left(\mu_{i t_{1}}, \cdots, \mu_{i t_{n}}\right)^{\prime}$, and $\mu_{i}=\partial \mu_{i} / \partial \beta$. In practice, $V_{i}$ is often unknown, and the empirical estimator of $V_{i}$ based on sample variance could be unreliable, especially when there is a small number of replications relative to a large number of variance components. Liang and Zeger (1986) introduced generalized estimating equations to simplify 
$V_{i}$ by assuming $V_{i}=A_{i}^{1 / 2} R A_{i}^{1 / 2}$, where $A_{i}$ is a diagonal marginal variance matrix and $R$ is a common working correlation which involves a small number of nuisance parameters. If the working correlation $R$ is misspecified, the estimator of the regression parameter is still consistent, but is not efficient within the same class of estimating functions.

Qu et al. (2000) introduced the quadratic inference function by assuming that the inverse of the working correlation can be approximated by a linear combination of several basis matrices, that is,

$$
R^{-1} \approx a_{0} I+a_{1} M_{1}+\ldots a_{m} M_{m}
$$

where $I$ is the identity matrix and $M_{i}$ are symmetric matrices. The advantage of this approach is that it does not require estimation of linear coefficients $a_{i}$ 's which can be viewed as nuisance parameters, since the generalized estimating equation is a linear combination of elements of the estimating functions

$$
\bar{g}_{N}(\beta)=\frac{1}{N} \sum_{i=1}^{N} g_{i}(\beta) \approx \frac{1}{N}\left(\begin{array}{c}
\sum_{i=1}^{N}\left(\dot{\mu}_{i}\right)^{\prime} A_{i}^{-1}\left(y_{i}-\mu_{i}\right) \\
\sum_{i=1}^{N}\left(\dot{\mu}_{i}\right)^{\prime} A_{i}^{-1 / 2} M_{1} A_{i}^{-1 / 2}\left(y_{i}-\mu_{i}\right) \\
\vdots \\
\sum_{i=1}^{N}\left(\dot{\mu}_{i}\right)^{\prime} A_{i}^{-1 / 2} M_{m} A_{i}^{-1 / 2}\left(y_{i}-\mu_{i}\right)
\end{array}\right)
$$

Because the dimension of the above estimating equations is greater than the number of unknown parameters, we cannot set each component in (2) to be zero to solve for $\beta$. Instead we estimate $\beta$ by setting $\bar{g}_{N}$ as close to zero as possible, in the sense of minimizing the quadratic function:

$$
\widehat{\beta}=\arg \min _{\beta} \bar{g}_{N}^{\prime} \Omega^{-1} \bar{g}_{N},
$$

where $\Omega=\operatorname{var}\left(g_{i}\right)$, here we assume $N$ subjects are independent and identically distributed. The estimator in (3) is also called generalized method of moments estimator in econometrics literature (Hansen, 1982). The covariance $\Omega$ is invertiable if estimating equations in $g_{i}$ are not linearly dependent. The covariance $\Omega$ in (3) is often unknown, but it can be estimated consistently by $\bar{C}_{N}=N^{-1} \sum g_{i} g_{i}^{\prime}$. Note the additional requirement for $\overline{C_{N}}$ being invertible is that $N \geq \operatorname{dim}\left(g_{i}\right)$. The quadratic function

$$
Q_{N}(\beta)=N \bar{g}_{N}^{\prime} \bar{C}_{N}^{-1} \bar{g}_{N}
$$

is called the quadratic inference function (Qu et al., 2000), because it provides an inference function for testing of $\beta$. This approach also provides an optimal linear combination of given estimating functions such that the asymptotic variance of the estimator attains the minimum in the sense of Löwner ordering (e.g., Pukelsheim, 1993, p. 12). 


\subsection{An effective estimation procedure via penalized quadratic inference functions}

Under the settings of generalized linear models, varying coefficient models assume that the following mean structure

$$
E\left\{y_{i}\left(t_{i j}\right) \mid x\left(t_{i j}\right)\right\}=h\left\{x_{i}^{\prime}\left(t_{i j}\right) \beta\left(t_{i j}\right)\right\}=\mu_{i j}
$$

where $h(\cdot)$ is a known inverse link function. Varying coefficient models were systematically introduced by Hastie and Tibshirani (1993). Here we are interested in the setting of longitudinal data where data are correlated within the same subject. Suppose $B_{u v}(t)$ is a set of basis functions of the functional space to which $\beta_{u}(\cdot)$ belongs, and $\beta_{u}(t)$ can be approximated by a linear combination of the basis functions. Specifically,

$$
\beta_{u}(t) \approx \sum_{v=0}^{V_{u}} \gamma_{u v} B_{u v}(t), \text { for } u=1, \cdots, p
$$

where $\gamma_{u v}$ 's are constants, and $V_{u}$ is associated with the number of basis functions for the $u$ th coefficient. Substituting the approximation of $\beta_{u}(t)$ into (5), the mean function in (5) can be approximated by

$$
h\left\{X_{i}^{\prime}\left(t_{i j}\right) \beta\left(t_{i j}\right)\right\} \approx h\left[\sum_{u=1}^{p} \sum_{v=0}^{V_{u}}\left\{x_{i u}\left(t_{i j}\right) B_{u v}\left(t_{i j}\right)\right\} \gamma_{u v}\right] .
$$

The basis functions can be selected as polynomials, Fourier basis functions or splines. Our approach is not restricted to one specific choice of basis functions, but here we consider only the $q$-degree truncated power spline basis with knots $\kappa_{1}, \cdots, \kappa_{K_{u}}$

$$
1, t, \cdots, t^{q},\left(t-\kappa_{1}\right)_{+}^{q}, \cdots,\left(t-\kappa_{K_{u}}\right)_{+}^{q},
$$

where $(z)_{+}^{q}=z^{q} I(z \geq 0)$. In Section 4, we will discuss how to choose $q$ and the number of knots using a goodness-of-fit test, and generalized method of moments model selection criterion. With the truncated power spline basis, the coefficient function can be modeled by

$$
\beta_{u}(t)=\gamma_{u 0}+\gamma_{u 1} t+\ldots+\gamma_{u q} t^{q}+\sum_{k=1}^{K_{u}} \gamma_{u(q+k)}\left(t-\kappa_{k}\right)_{+}^{q}
$$

To incorporate correlation into the model, we apply the idea of the quadratic inference function in Section 2.1 and construct estimating functions as follows. We create $\bar{g}_{N}$ as in (2) with the mean of response $\mu_{i}$ in (5) approximated by using the basis in (6). We can further derive a quadratic inference function $Q_{N}(\gamma)$ in (4), which is a function of parameters $\gamma=\left\{\gamma_{u v}, u=1\right.$, $\ldots, p ; v=0, \ldots, V_{u}$. Minimizing $Q_{N}(\gamma)$ yields an estimator for $\gamma$. Plugging the estimator of $\gamma_{u v}$ into the basis expansion (6), we obtain an estimator for $\beta_{u}(t)$. However, it is well known that the model in (6) usually over-parameterizes the coefficient function, and therefore the 
resulting estimator is undersmoothed and has a large variance. To overcome this drawback, we borrow the idea of the penalized spline (Ruppert and Carroll, 2000) and propose a penalized quadratic inference function:

$$
N^{-1} Q_{N}(\gamma)+\lambda \gamma^{\prime} D \gamma
$$

where $D$ is a diagonal matrix with 1 if $\gamma_{u v}$ is the coefficient of the truncated power function associated with the knots in (6), and 0 otherwise, and $\lambda$ is a smoothing parameter which can be chosen by data-driven methods such as cross-validation and generalized cross-validation. If $\lambda$ is large, it has more shrinkage towards a polynomial fit, less weights on selected knots, and it is oversmoothed. On the other hand, if $\lambda_{N}$ is small, it is undersmoothed. See Wand (1999),Ruppert (2002), Yu and Ruppert (2002),Kim, et al. (2003),Jarrow et al. (2004) and Yu and Ruppert (2004) on the penalized spline approach.

\subsection{Asymptotic properties of estimators}

In this section, we will study the asymptotic properties of the penalized quadratic inference function estimator. Here we focus only on fixed-knot asymptotics, since fixed knots spline regression might be more useful for developing a practical statistical methodology, as argued by Yu and Ruppert (2002). We first establish the asymptotic properties of the penalized quadratic inference function estimator when the smoothing parameter $\lambda_{N}$ goes to 0 as sample size $N$ goes to infinity. Theorem 1 shows the strong consistency of the resulting estimator of the penalized quadratic inference function, and Theorem 2 establishes the root $N$ consistency and asymptotic normality of the resulting estimator.

Theorem 1-Under Conditions A-D in the Appendix, if the smoothing parameter $\lambda_{N}=o(1)$, then the spline regression parameter estimator $\hat{\gamma}$ by minimizing (7) exists and converges to $\gamma_{0}$ almost surely.

Theorem 2-Under Conditions A-E in the Appendix, if the smoothing parameter $\lambda_{N}=o$ $\left(N^{-1 / 2}\right)$, then the spline regression parameter estimator $\hat{\gamma}$ by minimizing (7) is asymptotically normal and efficient (i.e., the asymptotic variance of the estimator reaches lower bound). That is

$$
\sqrt{N}\left(\widehat{\gamma}-\gamma_{0}\right) \stackrel{d}{\rightarrow} N_{k}\left(0,\left(J_{0}^{\prime} C_{0}^{-1} J_{0}\right)^{-1}\right),
$$

where $\gamma_{0}$ is the parameter satifying $E_{\gamma_{0}} g_{i}=0, k=\sum_{u=1}^{p} V_{u}+p, J_{0}$ and $C_{0}$ are given by $\frac{\partial \bar{g}_{N}}{\partial \gamma}$ converges to $J_{0}$ in probability and $\bar{C}_{N}=N^{-1} \sum g_{i} g_{i}^{\prime}$ converges to $C_{0}$ in probability.

(How to compare GEE? Could you please add some arguments on QIF is more efficient than GEE when working correlation structure is misspecified. I think, it will more convince the referees and AE why people should use QIF rather than GEE.)

Next, we establish the asymptotic distribution when $\lambda$ is treated as fixed. Notice that $\hat{\gamma}$ by minimizing (7) is asymptotically equivalent to solving

$$
J_{0}^{\prime}(\gamma) C_{0}^{-1}(\gamma) \bar{g}_{N}(\gamma)+\lambda D \gamma=0
$$


Let $s_{i}=J_{0}^{\prime} C_{0}^{-1} g_{i}+\lambda D \gamma$, then $\hat{\gamma}(\lambda)$ solves $\sum_{i=1}^{N} s_{i}\{\gamma(\lambda), \lambda\}=0$. If we assume that $E\left[s_{1}\{\gamma(\lambda), \lambda\}\right]=$ 0 for a fixed $\lambda$, then we are solving unbiased estimating equations. The following asymptotic distribution of $\hat{\gamma}(\lambda)$ can be derived based on estimating function theory (e.g. Heyde, 1997, Chap. 2):

$$
\sqrt{N}\{\widehat{\gamma}(\lambda)-\gamma(\lambda)\} \stackrel{d}{\rightarrow} N_{k}\left(0, H^{-1} G H^{-1}\right),
$$

where $H=E\left(\partial s_{1} / \partial \gamma\right)=J_{0}^{\prime} C_{0}^{-1} J_{0}+\lambda D$ and $G=E\left(s_{1} s_{1}^{\prime}\right)$.

\section{Practical implementation issues}

In practical implementation, one has to choose the basis for the inverse of the correlation matrix, determine the magnitude of $\lambda$, and calculate the standard error and confidence interval of the resulting estimator. In this section, we address these practical issues.

\subsection{Choice of the basis for the inverse of the correlation matrix}

We discuss the choice of basis matrices $M_{i}$ in (1) in this section. If the working correlation is exchangeable, we can choose a basis matrix $M_{1}$ with 0 on the diagonal and 1 off-diagonal. If the working correlation is AR-1, then $M_{1}^{*}$ can be 1 on the sub-diagonal and 0 elsewhere, and $M_{2}^{*}$ can be 1 on $(1,1)$ and $(N, N)$ components and 0 elsewhere. However, $M_{2}^{*}$ can often be dropped out of the model, as removing $M_{2}^{*}$ does not affect the efficiency of the estimator too much, but could simplify the estimation procedure. If we use both $M_{1}$ and $M_{1}^{*}$, then they are effective for modeling either the exchangeable or AR-1 working correlation. This is useful when there is uncertainty as to which working correlation structure is appropriate. Our simulation also confirms this finding. If there is no prior information on working correlation, $\mathrm{Qu}$ and Lindsay (2003) provide an adaptive estimation equation approach to approximate the true correlation empirically, their approach does not require to invert large dimensional unstructured correlation matrix.

\subsection{Choice of smoothing parameter}

Selection of the smoothing parameter is crucial in model fitting. It is desirable to have an automatic and data-driven method to select the smoothing parameter. Here we extend generalized cross-validation to the penalized quadratic inference function. Following the conventional technique of penalized least squares (e.g., Ruppert, 2002), we define the effective degrees of freedom as

$$
\mathrm{df}=\operatorname{trace}\left\{\left(\ddot{Q}_{N}+N \lambda D\right)^{-1} \ddot{Q}_{N}\right\}
$$

where $Q_{N}$ is the second derivative of $Q_{N}$ with respect to $\gamma$. Thus, a generalized cross-validation statistic is defined as

$$
\operatorname{GCV}(\lambda)=\frac{N^{-1} Q_{N}}{\left(1-N^{-1} \mathrm{df}\right)^{2}}
$$

and further $\hat{\lambda}=\operatorname{argmin}_{\lambda} \operatorname{GCV}(\lambda)$. In practice, the above minimization can be carried out by searching over a grid of $\lambda$ values. 


\subsection{Standard error formula}

We can derive the standard error formula for the resulting estimator using the sandwich formula,

$$
\widehat{\operatorname{cov}}(\gamma)=\left\{\ddot{Q}_{N}(\gamma)+N \lambda D\right\}^{-1}\left(\sum s_{i} s_{i}^{\prime}\right)\left\{\ddot{Q}_{N}(\gamma)+N \lambda D\right\}^{-1}
$$

which can be shown to be a consistent estimator of $\operatorname{cov}(\hat{\gamma})$ as given in Theorem 2. Denote $B_{u}(t)=\left[B_{u 1}(t), \cdots, B_{u V_{u}}(t)\right]^{\prime}$ and $\hat{\gamma}_{u}=\left[\hat{\gamma}_{u 1}, \cdots, \hat{\gamma}_{u V_{u}}\right]^{\prime}$. Then an estimator for $\beta_{u}(t)$ is $\widehat{\beta}_{u}(t)=B_{u}^{\prime}(t) \widehat{\gamma}_{u}$ and its covariance can be estimated by $\widehat{\operatorname{cov}}\left\{\widehat{\beta}_{u}(t)\right\}=B_{u}^{\prime}(t) \widehat{\operatorname{cov}}\left(\widehat{\gamma}_{u}\right) B_{u}(t)$.

Ignoring the approximation error in (6), a 100(1- $\alpha) \%$ pointwise confidence interval of $\beta_{u}(t)$ is given by

$$
\widehat{\beta}_{u}(t) \pm z_{\alpha / 2} \sqrt{\widehat{\operatorname{cov}}\left\{\widehat{\beta}_{u}(t)\right\}}
$$

where $z_{\alpha / 2}$ is the $100(1-\alpha / 2)$ th percentile of the standard normal distribution, since the resulting estimator follows an asymptotic normal distribution by Theorem 2 .

\section{Nonparametric goodness-of-fit tests and model selection}

In practice, parsimonious models are always desirable to enhance model predictability. It is of interest to test whether parsimonious parametric models can be used to approximate coefficient functions from the shape of their estimators. For varying coefficient models, it is of particular interest to test whether some coefficients are time-varying or time-invariant. In other words, we are interested in testing

$$
H_{0}: \beta_{u}(\cdot) \equiv \beta_{u 0} \text { verus } H_{1}: \beta_{u}(\cdot) \neq \beta_{u 0}
$$

for some $u$, where $\beta_{u 0}$ is an unknown constant. This problem can be handled using truncated power splines regression as in (6). Based on (6), we can test the following null hypothesis:

$$
H_{0}: \gamma_{u v}=0, v=1, \ldots, V_{u} .
$$

Let $\tilde{\gamma}$ denote the estimator under $H_{0}$ and $\hat{\gamma}$ be the estimator under $H_{1}$. Since the quadratic inference function plays a similar role of the least-square function, $Q(\tilde{\gamma})$ and $Q(\hat{\gamma})$ measure how well the model fits the data under $H_{0}$ and $H_{1}$, respectively. Intuitively, under $H_{0}$ the difference between $Q(\tilde{\gamma})$ and $Q(\hat{\gamma})$ should be very small. However, under $H_{1}, Q(\gamma)$ should be systematically larger than $Q(\hat{\gamma})$. Thus, an appropriate test statistic to test $H_{0}$ against $H_{1}$ would be

$$
T=Q(\tilde{\gamma})-Q(\hat{\gamma})
$$

Alternatively, if we apply the penalized quadratic inference function, we may also consider 


$$
T_{a}=Q(\tilde{\gamma})+N \lambda_{N} \tilde{\gamma}^{\prime} D \tilde{\gamma}-Q(\widehat{\gamma})-N \lambda_{N} \widehat{\gamma}^{\prime} D \widehat{\gamma}
$$

We next demonstrate that under certain regularity conditions and under $H_{0}, T$ and $T_{a}$ have the same limiting distribution, and both of them have a chi-squared distribution.

\section{Theorem 3}

Under conditions A-E in the Appendix, if the smoothing parameter $\lambda_{N}=o\left(N^{-1 / 2}\right)$, then $T$ and $T_{a}$ asymptotically follow chi-squared with degrees of freedom equal to $V_{u}$ under the null hypothesis in (11).

An important issue arises here as to how we decide the varying coefficient function in (6) is adequately modeled. To assess whether there is a sufficient number of basis functions in (6) such that the model assumption $E(g)=0$ is satisfied, where $g$ is the estimating function in (2) for a single observation, we apply the goodness-of-fit test (Hansen, 1982). Namely,

$$
Q(\widehat{\gamma}) \stackrel{d}{\rightarrow} \chi_{r-k}^{2}
$$

where $\hat{\gamma}$ is the estimator by minimizing the quadratic inference function when given basis functions are applied in the model, and $r$ is the dimension of $\bar{g}_{N}$ in (2), and $k$ is the dimension of $\gamma$. This test can also be useful to determine the number of knots to be selected in (6), as too many knots in the model might overfit the data and degrade the performance of spline estimators (Ruppert, 2002). Notice that the above goodness-of-fit test is only applicable where there are more estimating functions than unknown parameters. So it works for our situation as the dimension of estimating functions in (2) is greater than the dimension of parameters.

It is also possible that the goodness-of-fit tests fail to reject several different models. How can we assess which model is better? Notice that most of these models are not nested since different models likely have different knots, here we let knots be equally spaced. Andrews (1999) proposed model selection or moment selection criterion in the generalized method of moments framework, which can also be applied here. The main idea is to penalize the objective function $Q(\hat{\gamma})$ for the difference of the numbers of estimating equations and parameters. For example, Andrew's selection criterion for a model with $r$ estimating equations and $k$ parameters is

$$
Q(\widehat{\gamma})-(r-k) c_{N}
$$

where $c_{N}$ is $\ln N$ for BIC and 2 for AIC, which are commonly used in traditional BIC and AIC model selection criterion. A model with a smaller value in (13) is better. The BIC in general is better than the AIC as the latter is not consistent for different sample size. Intuitively, the penalty term associated with $r-k$ can be explained that $Q(\hat{\gamma})$ follows asymptotically $\chi_{r-k}^{2}$, and the mean of the chi-squared is its degrees of freedom. In our setting, if we choose $m+1$ bases matrices as in (2), then $r-k=(m+1) k-k=m k$, that is, we penalize models with too many parameters associated with high degree of polynomial functions and large number of knots.

For the multivariate model with several varying coefficients, we could set the upper limit of knots as 20 (Ruppert, 2002), and $q$ as 5. ( $t$ ) In practice, these numbers could be reduced to be 10 for the number of knots and 3-degree polynomial basis functions. This is because that in principle, choice of basis functions does not affect the fit very much, although some bases 
functions are more numerically stable with simpler computation (Ruppert et al., 2003, p. 69). After we determine these, we could choose the optimal combination of knots and basis functions where the BIC is the minimum. The selected model with the minimum BIC provides the best fit of the data within a class where upper limit number of knots and the degrees of polynomial basis functions are determined. The model selection procedure could be computationally intensive (Ruppert et al., 2003, p. 64) as it is possible that the total number of combination is very high. Wand (2000) provides review and comparison for some recent model selection approaches.

It is important to point out that model selection should be done at the begining stage since the hypothesis testing for whether the coeficient is time varying or not depends on how we choose the full model. If the full models under the alternative are different, then test statistics and corresponding $p$-values could be different, although it might not affect statistical significance of our tests dramatically.

\section{Simulation and application}

In this section, we assess the finite sample performance of the proposed procedures in Sections 2 and 3 with Monte Carlo simulation studies. We also demonstrate the proposed method by an analysis of an AIDS data set.

\subsection{Simulation studies}

Example 1 (Binary response)—We generate 200 subjects for each simulation. Each subject is supposed to have 31 repeated measurements at centered scheduled time points $\{-15$, $-14, \ldots, 15\}$, but in reality each subject has a $60 \%$ chance of missing the scheduled time except at the beginning time. The true time also varies around the unskipped schedule time following the uniform $(-0.5,0.5)$ distribution. The response variable $y_{i j}$ has the marginal distribution

$$
P\left(y_{i j}=1 \mid t_{i j}\right)=\exp \left\{\beta\left(t_{i j}\right)\right\} /\left[1+\exp \left\{\beta\left(t_{i j}\right)\right\}\right],
$$

where $i=1, \ldots, 200$ and $j=1, \ldots, n_{i}$. We simplify this simulation by assuming that covariates are constant 1 . We model 4 kinds of varying coefficients $\beta(t)$ as follows:

$$
\begin{aligned}
& \beta_{0}(t)=\sin \left(\frac{(t+15) \pi}{60}\right)-0.5, \quad \beta_{1}(t)=\cos \left\{\frac{(t+10) \pi}{15}\right\} \\
& \beta_{2}(t)=-0.1(t-15), \quad \beta_{3}(t)=\frac{(5-t)^{3}}{4000}-1 .
\end{aligned}
$$

To create correlated responses, we apply the algorithm following Park et al. (1996) under exchangeable correlation structure with the correlation parameter as 0.5 .

We first perform the goodness-of-fit test in Section 4 to select the degree of truncated power polynomial splines and the number of knots which are evenly distributed over the ranges of $t_{i j}$. The results are summarized as follows. For basis function in (6), the goodness-of-fit test yields $q=3$ for $\beta_{0}(t), \beta_{1}(t)$ and $\beta_{3}(t)$, and $q=2$ for $\beta_{2}(t)$; for number of knots, the test also chooses 5 knots for $\beta_{1}(t)$, and no knots for $\beta_{0}(t), \beta_{2}(t)$ and $\beta_{3}(t)$. With the selected degrees and the number of knots, we calculate the quadratic inference function estimators by minimizing (7). Here we assume exchangeable working correlation for estimating equations in (2), that is, the basis matrix $M_{1}$ is 0 on the diagonal and 1 off-diagonal. The result by assuming AR-1 working correlation is similar to that under exchangeable correlation, and is not presented here. We define the mean absolute deviation of errors by minimizing (7) 


$$
\operatorname{MADE}=\sum_{j=0}^{30} 31^{-1} \widehat{\mathcal{\beta}}\left(t_{j}\right)-\beta\left(t_{j}\right) \mid / \operatorname{range}(\beta),
$$

where $t_{j}=(-15, \ldots, 15)$. Figure 1 provides fitted varying coefficient curves corresponding to nine deciles of mean absolute deviation of errors from 1000 simulations. Figure 1 demonstrates that the quadratic inference function approach applying the penalized spline works well in settings where coefficients have linear, cubic, sine and cosine relationships of time.

We apply the test result in (12) to illustrate how the quadratic inference function performs for testing whether coefficients vary over time in finite samples. We simulate data such that $\beta_{1}(t)$ $=0.5$. The null hypothesis $H_{0}: \beta_{1}$ is constant over time. We let the basis functions for $\beta_{1}(t)$ under $H_{0}$ be 1 and basis functions for $\beta_{1}(t)$ under the alternative be $1, t, t^{2}, t^{3}$, $(t+10)_{+}^{3},(t)_{+}^{3},(t-10)_{+}^{3}$. We calculate $\tilde{\gamma}_{1}$ and $\hat{\gamma}_{1}$ by minimizing $(4)$, where $\bar{g}_{N}$ is constructed by assuming either exchangeable or AR-1 working correlation structures. Since the difference of the numbers for the basis functions under $H_{1}$ and $H_{0}$ is 6 , the test statistic $Q(\tilde{\gamma})-Q(\hat{\gamma})$

asymptotically follows $\chi_{6}^{2}$. Figure 2 provides quantile-quantile plots under both exchangeable and AR-1 working correlations and illustrates that under $H_{0}$ the empirical quantiles of $Q(\tilde{\gamma})-$ $Q(\hat{\gamma})$ follow the theoretical chi-squared quantile rather well.

We next examine the power of the quadratic inference function approach when $\beta_{1}(t)$ deviates from the constant. Let

$$
\beta_{1}(t, \eta)=0.5-\eta * \cos \left\{\frac{(t-10) \pi}{15}\right\}
$$

where $0 \leq \eta \leq 1$. We calculate test statistics $Q(\tilde{\gamma})-Q(\hat{\gamma})$ from 1000 simulations for various $\eta$, and find the percentage of test statistics greater to or equal to 12.59 , the $95 \%$ quantile of $\chi_{6}^{2}$. Figure 3 illustrates the power function curve. Notice that when $\eta$ is close to 0 , the test size is approximately 0.05 ; and when $\eta$ reaches 0.25 , the probability of rejection reaches 1 .

Example 2 (Continuous response)-In this example, we generate 200 subjects, and each subject is supposed to have repeated measurements at scheduled time points $\{0,1 \ldots, 30\}$, but has a $60 \%$ chance of missing the scheduled time except at time 0 . The true time also varies around the unskipped schedule time following the uniform $(-0.5,0.5)$ distribution. The response variable $y_{i j}$ is modeled as

$$
y_{i j}=\beta_{0}\left(t_{i j}\right)+\sum_{k=1}^{3} X_{i}^{(k)}\left(t_{i j}\right) \beta_{k}\left(t_{i j}\right)+\varepsilon_{i}\left(t_{i j}\right),
$$

where $i=1, \ldots, 200$ and $j=1, \ldots, n_{i}$. The time-varying coefficients satisfy:

$$
\begin{array}{ll}
\beta_{0}(t)=15+20 \sin \left(\frac{t \pi}{60}\right), & \beta_{1}(t)=2-3 \cos \left\{\frac{(t-25) \pi}{15}\right\} \\
\beta_{2}(t)=6-0.2 t, & \beta_{3}(t)=-4+\frac{(20-t)^{3}}{1000} .
\end{array}
$$


The covariates are generated as follows: $X_{i}^{(1)}$ has a uniform $(t / 10,2+t / 10)$ distribution; conditioning on $X_{i}^{(1)}, X_{i}^{(2)}$ has a normal distribution with mean 0 and variance $\left(1+X_{i}^{(1)}\right) /\left(2+X_{i}^{(1)}\right)$; and $X_{i}^{(3)}$ has a Bernoulli(0.6) distribution. The error $\varepsilon_{i}$ follows a multivariate normal distribution with mean 0 and the marginal variance matrix $2 I, I$ is an identity matrix, and the correlation is exchangeable with correlation 0.8 .

We select the degrees $q$ and the number of knots using the goodness-of-fit test in Section 4 . The test provides $q=3$ for all coefficients, 5 knots for $\beta_{1}(t)$ and no knots for $\beta_{0}(t), \beta_{2}(t)$ and $\beta_{3}(t)$. With the selected degrees and the number of knots, we calculate the quadratic inference function estimators by minimizing (7) with exchangeable working correlation structure.

As in Example 1, Figure 4 provides fitted varying coefficient curves corresponding to nine deciles of mean absolute deviation of errors from 1000 simulations. Figure 4 clearly shows that the quadratic inference function approach applying the penalized spline works well in settings where coefficients have linear, cubic, sine and cosine relationships of time.

We also use this simulation to illustrate how different working correlation could affect our estimations. The true correlation is exchangeable here, we calculate the quadratic inference function estimators by minimizing (7) using AR-1 working correlation and independent structures in addition to above exchangeable correlation structure, we caculate the median of absolute deviation of errors between the fitted and true values of $\beta_{0}, \beta_{1}, \beta_{2}$ and $\beta_{3}$, and plot them against time $t=0,1, \ldots, 30$. Figure 6 shows that the estimation based on independent structure has the largest median of absolute deviation of errors for every time point. Estimations using AR-1 and exchangeable have similar errors, though using the true exchangeable correlation structure produces least median of absolute deviation of errors.

To illustrate how the quadratic inference function performs for testing whether coefficients vary over time in finite samples, we simulate the same data as above, except let $\beta_{1}(t)=2$ such that under the null hypothesis $H_{0}: \beta_{1}$ is constant over time. We let the basis functions for $\beta_{1}$ under $H_{0}$ be 1 , and the basis functions for $\beta_{1}(t)$ under the alternative be $1, t, t^{2}, t^{3}$,

$(t-5)_{+}^{3},(t-10)_{+}^{3},(t-15)_{+}^{3},(t-20)_{+}^{3},(t-25)_{+}^{3}$. We calculate $\tilde{\gamma}_{1}=\tilde{\gamma}_{10}$ and $\hat{\gamma}_{1}=\left(\hat{\gamma}_{10}, \hat{\gamma}_{11}, \ldots, \hat{\gamma}_{18}\right)$ by minimizing (4), where $\bar{g}_{N}$ is constructed by assuming either exchangeable or AR-1 working correlation structures. Since the difference of the numbers for the basis functions under $H_{1}$ and $H_{0}$ is 8 , the test statistic $Q(\tilde{\gamma})-Q(\hat{\gamma})$ asymptotically follows $\chi_{8}^{2}$. Figure 5 provides quantilequantile plots under exchangeable and AR-1 working correlation and illustrates that under $H_{0}$ the empirical quantiles of $Q(\tilde{\gamma})-Q(\hat{\gamma})$ follow the theoretical chi-squared quantile rather well.

We also examine the power of the quadratic inference function approach when $\beta_{1}(t)$ deviates from the constant. Let

$$
\beta_{1}(t, \eta)=2-\eta * 3 \cos \left\{\frac{(t-25) \pi}{15}\right\}
$$

where $0 \leq \eta \leq 1$. We calculate test statistics $Q(\tilde{\gamma})-Q(\hat{\gamma})$ from 1000 simulations for various $\eta$ using exchangeable, AR-1 correlation and independent structures, and find the percentage of test statistics greater or equal to 15.51 , the $95 \%$ quantile of $\chi_{8}^{2}$. Figure 7 illustrates power function curves for three correlation structures. Notice that when $\eta$ is close to 0 , the test sizes are all approximately 0.05 ; and when $\eta$ reaches 0.06 , the probabilities of rejection reach 1 . In addition, the power function using true exchangeable correlation structure is much higher than 
the power using AR-1 and independent structures when $\eta<0.06$. The test power using AR-1 and independent structures are close, however, the test power by assuming independent structure is the worst.

\subsection{Application to AIDS data}

We apply AIDs data (Huang et al., 2002) to illustrate how the penalized quadratic inference function approach works for real data. This data set consists of 283 homosexual males who were HIV positive between 1984 and 1991. Each patient was supposed to have measurements taken every 6 months, but it often happened that patients missed appointments or rescheduled their appointments. Therefore each patient had a different number of repeated measurements and the true observation times were not equally spaced. Here time $t$ is defined as the time (in years) when subjects had their visits after their HIV infection. We observe that each patient has minimum 1 and maximum 14 measurements for this data. It is known that HIV destroys CD4 cells, so by measuring CD4 cell counts and percentages in the blood, doctors are able to monitor progression of the disease. The response variable is the CD4 percentage over time. Three covariates were also collected: patient's age, smoking status with 1 as smoker and 0 as nonsmoker, and the CD4 cell percentage before their infection. We model it as

$$
y_{i j}=\beta_{0}\left(t_{i j}\right)+\beta_{1}\left(t_{i j}\right) \text { Smoke }+\beta_{2}\left(t_{i j}\right) \text { Age }+\beta_{3}\left(t_{i j}\right) \text { Pre }-\mathrm{CD} 4+\varepsilon_{i j} .
$$

We perform the goodness-of-fit test and choose $q=3$ in the basis functions of (6) for all coefficients, and the number of knots to be $0,5,1$, and 3 for $\beta_{u}(t), u=0,1,2,3$ respectively.

Figure 8 provides fitted curves (the solid line) for coefficients of intercept, smoking, age and pre-infection CD4 effects. We also provide $95 \%$ pointwise confidence intervals (dotted lines) for four varying coefficients. Figure 8 also implies that the intercept decreases over time, though the rate of decreasing drops; smoking and age effects are not significant; and the pre-infection CD4 percentage appears to have a positive relationship with the post-infection CD4 percentage. These findings are consistent with Wu and Chiang (2000),Fan and Zhang (2000) and Huang et al. (2002).

To quantify these findings, we apply the quadratic inference function test in (12) to test whether smoking and age effects are statistically significant. For intercept and pre-infection CD4 effects, we also test whether they are time-invariant or not. We fit the model with a 3-degree polynomial function of $t$ for all varying coefficients, a total of 25 parameters with 4 ( 0 knots) for intercept, 9 (5 knots) for smoking, 5 (1 knot) for age and 7 (3 knots) for pre-infection CD4. We assume an exchangeable working correlation, therefore the number of estimating equations $r=25 \times 2=50$. The goodness-of-fit test statistic $Q(\tilde{\gamma})=23.1$. By Section 4, with $r-k=25$ degrees of freedom, the asymptotic $p$-value from the chi-squared test is 0.57 . This indicates that the model fits the data reasonably well based on the asymptotic results in Section 4. For testing whether smoking is significant, we set all 9 parameters for the smoking coefficient to be 0 , and estimate the rest of the 16 parameters by minimizing the quadratic inference function in (7). The test statistic under $H_{0}$ is 36.1 , the difference between the two test statistics under $H_{1}$ and $H_{0}$ is then 13.0, and the corresponding $p$-value is 0.163 based on chi-squared with 25 $-16=9$ degrees of freedom. Similarly, we obtain the $p$-value for age as 0.172 . None of these are statistically significant.

To test whether the intercept effect is time-varying, we set the 3 parameters associated with time $t$ to be 0 (the intercept coefficient has no knots, so there is a total of 4 parameters). The test statistic under $H_{0}$ is 105.0, the difference between the test statistics under $H_{0}$ and $H_{1}$ is 81.9 , and the corresponding $p$-value is close to 0 based on the $\chi_{3}^{2}$ test, where the degrees of 
freedom is calculated by $25-22=3$. Similarly, we find the $p$-value for constant pre-infection CD4 effects is 0.045 , which is statistically significant. The final result for pre-infection CD4 effects differs from that in Huang et al.'s (2002) bootstrap approach, where their bootstrap approximate $p$-value is 0.059 . Examination of Figure 8 on pre-infection CD4 effects favors our conclusion. Table 1 provides comparisons of $p$-values based on Huang et al.'s (2002) approach and our method. From Table 1, our $p$-values calculated from quadratic inference function test statistics are consistently smaller than Huang et al.'s results, this might be explained by that the quadratic inference function test takes into account the correlation information and thus may be more powerful.

\section{Discussion}

We propose nonparametric modeling using the penalized quadratic inference function to incorporate correlation for longitudinal data. Our approach works for continuous and discrete cases as it only requires correct specification of the mean structure which is modeled nonparametrically, and we do not require any likelihood or an approximation of the likelihood function for estimations and hypothesis testings such as in generalized linear mixed model approach. The quadratic inference function approach is relatively simple and numerically feasible since it does not involve any nuisance parameters associated with the working correlation. This advantage becomes more important in nonparametric settings as there are many more parameters involved in nonparametric modeling than in parametric or semiparametric modeling. Existing smoothing spline methods is mainly on correlated continuous responses. For correlated discrete data, existing nonparametric approaches either ignore correlation and treat data as independent, or could be computationally intensive and complex. For example, the generalized linear mixed effects model typically has parametric model assumption on random effect, and it requires numerical approximation to compute high dimensional likelihood functions even under the assumption that the random effests are normal. Estimation and hypotheses testing for variance components in generalized linear mixed effects are even more complicated if the random effects are not normal.

Another advantage of the quadratic inference function approach is that the inference function has an explicit asymptotic form, which allows us to test whether coefficients are time-varying or time-invariant for varying coefficient models. Further, it also enables us to do goodness-offit tests for checking model assumptions, and provides an objective criterion for choosing a sufficient number of basis functions and knots for varying coefficients. An important issue arised here is how to select basis functions and number of knots when candidate models are not nested to each other. This could be done using cross-validation and Mallows' (1973) $C_{p}$ criterion if the response is continuous normal (e.g., Ruppert et al., 2003). In the setting where there are more estimating equations than parameters such as in our case, we apply Andrew's (1999) generalized method of moments BIC criterion for selecting nonparametric basis functions and knots. This model selection criterion can be applied for both continuous and discrete responses.

\section{Acknowledgements}

The authors are grateful to many constructive suggestions from two referees, the Associate Editor and the Co-Editor. Qu's research was supported in part by an NSF grant DMS 0348764. Li's research was supported by a National Institute on Drug Abuse (NIDA) Grant 2-P50-DA10075.

\section{References}

Andrews DWK. Consistent moment selection procedures for generalized method of moments estimation. Econometrica 1999;67:543-564.

Billingsley, P. Probability and Measure. Vol. 3. New York: John Wiley \& Sons; 1995. 
Chiang CT, Rice JA, Wu CO. Smoothing spline estimation for varying coefficient models with repeatedly measured dependent variables. Journal of the American Statistical Association 2001;96:605-619.

Crainiceanu C, Ruppert D. Likelihood ratio tests in linear mixed models with one variance component. Journal of the Royal Statistical Society, Series B 2004;66:165-185.

Crowder M. On the use of a working correlation matrix in using generalised linear models for repeated measures. Biometrika 1995;82:407-410.

Fan J, Zhang JT. Functional linear models for longitudinal data. Journal of the Royal Statistical Society, Series B 2000;62:303-322.

Godambe VP. An optimum property of regular maximum likelihood estimation. Annals of Mathematical Statististics 1960;31:1208-1212.

Hansen L. Large sample properties of generalized method of moments estimators. Econometrica 1982;50:1029-1054.

Hart, JD. Nonparametric smoothing and lack-of-fit tests. New York: Springer; 1997.

Hastie TJ, Tibshirani RJ. Varying-coefficient models. Journal of the Royal Statistical Society, Series B 1993;55:757-776.

Heyde, CC. Quasi-likelihood and its application. New York: Springer-Verlag; 1997.

Hoover DR, Rice JA, Wu CO, Yang LP. Nonparametric smoothing estimates of time-varying coefficient models with longitudinal data. Biometrika 1998;85:809-822.

Huang JZ, Wu CO, Zhou L. Varying-coefficient models and basis function approximations for the analysis of repeated measurements. Biometrika 2002;89:111-128.

Jarrow R, Ruppert D, Yu Y. Estimating the interest rate term structure of corporate debt with a semiparametric penalized spline model. Journal of American Statistical Association 2004;99:57-66.

Kim I, Cohen N, Carroll RJ. Semiparametric regression splines in matched case-control studies. Biometrics 2003;59:1158-1169. [PubMed: 14969497]

Liang KY, Zeger SL. Longitudinal data analysis using generalised linear models. Biometrika 1986;73:1222.

Lin X, Carroll RJ. Nonparametric function estimation for clustered data when the predictor is measured without/with error. Journal of Computational and Graphical Statistics 2000;95:520-534.

Lin X, Wang N, Welsh AH, Carroll RJ. Equivalent kernels of smoothing splines in nonparametric regression for clustered/longitudinal data. Biometrika 2004;91:177-193.

Lipsitz SR, Molengberghs G, Fitzmaurice GM, Ibrahim J. GEE with Gaussian estimation of the correlations when data are incomplete. Biometrics 2000;56:528-536. [PubMed: 10877313]

Martinussen T, Scheike TH. Sampling adjusted analysis of dynamic additive regression models for longitudinal data. Scandinavian Journal of Statististics 2001;28:303-323.

Opsomer J, Wang Y, Yang Y. Nonparametric regression with correlated errors. Statistical Science 2001;16:134-153.

Park CG, Park T, Shin DW. A simple method for generating correlated binary variates. The American Statistician 1996;60:306-310.

Pukelsheim, F. Optimal Design of Experiments. New York: John Wiley \& Sons; 1993.

Qu A, Lindsay BG. Building adaptive estimating equations when inverse-of-covariance estimation is difficult. Journal of the Royal Statistical Society, Series B 2003;65:127-142.

Qu A, Lindsay BG, Li B. Improving generalised estimating equations using quadratic inference functions. Biometrika 2000;87:823-836.

Ruppert D. Selecting the number of knots for penalized splines. Journal of Computational and Graphical Statistics 2002;11:735-757.

Ruppert D, Carroll RJ. Spatially-adaptive penalties for spline fitting. Australian \& New Zealand Journal of Statistics 2000;42:205-223.

Ruppert, D.; Wand, MP.; Carroll, RJ. Semiparametric regression. New York: Cambridge University Press; 2003.

Wand MP. On the optimal amount of smoothing in penalized spline regression. Biometrika 1999;86:936940. 
Wand MP. A comparison of regression spline smoothing procedures. Computational Statistics 2000;15:443-462.

Wang N. Marginal nonparametric kernel regression accounting for within-subject correlation. Biometrika 2003;90:43-52.

Wang N, Carroll R, Lin X. Efficient semiparametric marginal estimation for longitudinal/cluster data. Journal of the American Statistical Association 2005;100:147-157.

Wang Y. Mixed-effects smoothing spline ANOVA. Journal of the Royal Statistical Society, Series B 1998a;60:159-174.

Wang Y. Smoothing spline models with correlated random errors. Journal of the American Statistical Association 1998b;93:341-348.

Wedderburn RWM. Quasi-likelihood functions, generalised linear models, and the Gauss-Newton method. Biometrika 1974;61:439-447.

Wu CO, Chiang CT, Hoover DR. Asymptotic confidence regions for kernel smoothing of a varyingcoefficient model with longitudinal data. Journal of the American Statistical Association 1998;93:1388-1402.

Wu CO, Chiang CT. Kernel smoothing on varying coefficient models with longitudinal dependent variable. Statistica Sinica 2000;10:433-456.

Yu Y, Ruppert D. Penalized spline estimation for partially linear single-index models. Journal of the American Statistical Association 2002;97:1042-1054.

Yu Y, Ruppert D. Root-n consistency of penalized spline estimator for partially linear single-index models under general Euclidean space. Statistica Sinica 2004;14:449-456.

Zhang D. Generalized linear mixed models with varying coefficient for longitudinal data. Biometrics 2004;60:8-15. [PubMed: 15032768]

\section{Appendix}

\section{Regularity conditions}

To establish the root $n$ consistency and asymptotic normality for the penalized quadratic inference function estimator, we need the following regularity conditions. Similar conditions are also given in Hansen (1982).

A. The weighting matrix $\bar{C}_{N}=N^{-1} \sum g_{i} g_{i}^{\prime}$ converges almost surely to a constant matrix $C_{0}$, where $C_{0}$ is invertible. This condition holds based on the weak law of large number when $N$ goes to infinity and the maximum cluster size is fixed.

B. The spline regression parameter $\gamma$ is identified, that is, there is a unique $\gamma_{0} \in S$ satisfying mean zero model assumption

$$
E\left\{g_{1}\left(\gamma_{0}\right)\right\}=0,
$$

where $S$ is the parameter space.

C. The parameter space $S$ is compact, and $\gamma_{0}$ is an interior point of $S$.

D. We require that $E\{g(\gamma)\}$ is continuous in $\gamma$.

E. The first derivative of $\bar{g}_{N}$ exists and is continuous, and $\frac{\partial \bar{z}_{N}}{\partial \gamma}(\widehat{\gamma})$ converges in probability to $J_{0}=E\left[\frac{\partial g}{\partial \gamma}\left(\gamma_{0}\right)\right]$ when $\hat{\gamma}$ converges in probability to $\gamma_{0}$.

\section{Proof of Theorem 1}

First, the estimator $\hat{\gamma}$ by minimizing (7) exists since (7) has 0 as a lower bound and the global minimum exists. Second, we will show that it is impossible that $\hat{\gamma}$ remains outside of $U$, where 
$U$ is any neighborhood of $\gamma_{0}$. First, $U^{c}$ is compact and $\left|C_{0}^{-1 / 2} E[g(\gamma)]\right|^{2}$ is continuous, where | $\left.\right|^{2}$ is the inner product of a vector. Following Conditions $C$ and $\mathrm{D},\left|C_{0}^{-1 / 2} E[g(\gamma)]\right|^{2}$ achieves a minimum in $U^{c}$, and let $\gamma^{*}$ be its minimum in $U^{c}$. Second, using identification of $\gamma$ from

Condition $\mathrm{B}$, we have $\left|C_{0}^{-1 / 2} E\left[g\left(\gamma^{*}\right)\right]\right|^{2}>0$. Hence, if we show $\left|C_{0}^{-1 / 2} E[g(\widehat{\gamma})]\right|^{2} \rightarrow_{a . s .} 0$, then eventually $\hat{\gamma} \in U$.

Since $\hat{\gamma}$ is the minimizer of (7), then

$$
\left|\bar{C}_{N}^{1 / 2} \frac{1}{N} \sum_{i=1}^{N} g_{i}(\widehat{\gamma})\right|+\lambda_{N} \widehat{\gamma}^{\prime} D \widehat{\gamma} \leq\left|\bar{C}_{N}^{-1 / 2} \frac{1}{N} \sum_{i=1}^{N} g_{i}\left(\gamma_{0}\right)\right|+\lambda_{N} \gamma_{0}^{\prime} D \gamma_{0}
$$

The right hand side of (15) converges to zero almost surely since $\lambda_{N}=o(1)$, and by the strong law of large numbers and Condition $\mathrm{A}$, that is,

$$
\left|\bar{C}_{N}^{-1 / 2} \frac{1}{N} \sum_{i=1}^{N} g_{i}\left(\gamma_{0}\right)\right| \rightarrow_{\text {a.s. }}\left|C_{0}^{-1 / 2} E\left[g\left(\gamma_{0}\right)\right]\right|^{2}=0
$$

Since $S$ is compact, we apply the uniform law of large numbers or Glivenko-Cantelli Theorem (Billingsley, 1995, p. 269),

$$
\sup _{b \in S}\left|\frac{1}{N} \sum_{i=1}^{N} g_{i}(b)-E[g(b)]\right| \rightarrow_{a . s .} 0
$$

Then by Condition A and the continuity mapping theorem,

$$
\left|\bar{C}_{N}^{-1 / 2} \frac{1}{N} \sum_{i=1}^{N} g_{i}(\widehat{\gamma})-C_{0}^{-1 / 2} E[g(\widehat{\gamma})]\right| \rightarrow_{a . s .} 0
$$

From (15), it follows that

$$
\left|C_{0}^{-1 / 2} E[g(\widehat{\gamma})]\right|^{2} \rightarrow \text { a.s. } 0
$$

as was to be shown.

\section{Proof of Theorem 2}

We denote $\dot{Q}$ and $Q \ddot{\text { to }}$ be the first and second derivative of the quadratic inference function $Q$ with respect to $\gamma$. Using the Central Limit Theorem, 


$$
\sqrt{N} \bar{g}_{N}\left(\beta_{0}\right) \stackrel{d}{\rightarrow} N_{r}\left(0, C_{0}\right)
$$

Since $\hat{\gamma}$ is obtained by minimizing (7), $\hat{\gamma}$ satisfies

$$
N^{-1} \dot{Q}(\gamma)+2 \lambda_{N} D \widehat{\gamma}=0
$$

By Taylor's expansion,

$0=N^{-1} \dot{Q}(\widehat{\gamma})+2 \lambda_{N} D \widehat{\gamma}=N^{-1} \dot{Q}\left(\gamma_{0}\right)+2 \lambda_{N} D \gamma_{0}+\left\{N^{-1} \ddot{Q}(\tilde{\gamma})+2 \lambda_{N} D\right\}\left(\widehat{\gamma}-\gamma_{0}\right)$,

where $\tilde{\gamma}$ is between $\gamma_{0}$ and $\hat{\gamma}$. Therefore

$$
\widehat{\gamma}-\gamma_{0}=\left\{N^{-1} \ddot{Q}(\tilde{\gamma})+2 \lambda_{N} D\right\}^{-1}\left\{N^{-1} \dot{Q}\left(\gamma_{0}\right)+2 \lambda_{N} D \gamma_{0}\right\}
$$

Notice that $N^{-1} \ddot{Q}(\tilde{\gamma})$ converges in probability to $2 J_{0}^{\prime}\left(\gamma_{0}\right) C_{0}^{-1}\left(\gamma_{0}\right) J_{0}\left(\gamma_{0}\right)=O_{p}(1)$ since $\tilde{\gamma}$ is between $\gamma_{0}$ and $\hat{\gamma}$, where $\hat{\gamma}$ converges to $\gamma_{0}$ in probability by Theorem 1 , and by Conditions $\mathrm{A}$ and $\mathrm{E}$. In addition, using the fact that $2 \lambda_{N} D=o\left(N^{-1 / 2}\right)$, therefore

$\left\{N^{-1} \ddot{Q}(\tilde{\gamma})+2 \lambda_{N} D\right\}^{-1}=2\left(J_{0}^{\prime} C_{0}^{-1} J_{0}\right)^{-1}+o_{p}\left(N^{-1 / 2}\right)$. Similarly,

$N^{-1} \dot{Q}\left(\gamma_{0}\right)+2 \lambda_{N} D \gamma_{0}=J_{0}^{\prime} C_{0}^{-1} \bar{g}_{N}\left(\gamma_{0}\right)+o\left(N^{-1 / 2}\right)$. Therefore equation (18) becomes

$$
\sqrt{N}\left(\widehat{\gamma}-\gamma_{0}\right)=\sqrt{N}\left(J_{0}^{\prime} C_{0}^{-1} J_{0}\right)^{-1}\left(J_{0}^{\prime} C_{0}^{-1} \bar{g}_{N}\right)+o_{p}(1) .
$$

Then using (16),

$$
\sqrt{N}\left(\gamma-\gamma_{0}\right) \stackrel{d}{\rightarrow} N_{p}\left(0,\left(J_{0}^{\prime} C_{0}^{-1} J_{0}\right)^{-1}\right)
$$

Next we will show that $\left(J_{0}^{\prime} C_{0}^{-1} J_{0}\right)^{-1}$ reaches minimum in Löwner ordering. Suppose we minimize $\bar{g}_{N}^{\prime} C^{-1} \bar{g}_{N}$, where $C$ is any arbitrary symmetric invertible matrix. Again, using the above arguments, the asymptotic $\operatorname{var}(\sqrt{N} \gamma)=\left(J_{0}^{\prime} C^{-1} J_{0}\right)^{-1}\left(J_{0}^{\prime} C^{-1} C_{0} C^{-1} J_{0}\right)\left(J_{0}^{\prime} C^{-1} J_{0}\right)^{-1}$.

Let $D=\left(J_{0}^{\prime} C^{-1} J_{0}\right)^{-1} J_{0}^{\prime} C^{-1} C_{0}^{1 / 2}-\left(J_{0}^{\prime} C_{0}^{-1} J_{0}\right)^{-1} J_{0}^{\prime} C_{0}^{-1 / 2}$,

$$
\begin{aligned}
& D D^{\prime}=\left(J_{0}^{\prime} C^{-1} J_{0}\right)^{-1} J_{0}^{\prime} C^{-1} C_{0} C^{-1} J_{0}\left(J_{0}^{\prime} C^{-1} J_{0}\right)^{-1}-\left(J_{0}^{\prime} C^{-1} J_{0}\right)^{-1} J_{0}^{\prime} C^{-1} J_{0}\left(J_{0}^{\prime} C_{0}^{-1} J_{0}\right)^{-1} \\
& -\left(J_{0}^{\prime} C_{0}^{-1} J_{0}\right)^{-1} J_{0}^{\prime} C^{-1} J_{0}\left(J_{0}^{\prime} C^{-1} J_{0}\right)^{-1}+\left(J_{0}^{\prime} C_{0}^{-1} J_{0}\right)^{-1} J_{0}^{\prime} C_{0}^{-1} J_{0}\left(J_{0}^{\prime} C_{0}^{-1} J_{0}\right)^{-1} \\
& =\left(J_{0}^{\prime} C^{-1} J_{0}\right)^{-1} J_{0}^{\prime} C^{-1} C_{0} C^{-1} J_{0}\left(J_{0}^{\prime} C^{-1} J_{0}\right)^{-1}-\left(J_{0}^{\prime} C_{0}^{-1} J_{0}\right)^{-1}
\end{aligned}
$$


Since $D D^{\prime}$ is nonegative definite matrix,

$\left(J_{0}^{\prime} C^{-1} J_{0}\right)^{-1} J_{0}^{\prime} C^{-1} C_{0} C^{-1} J_{0}\left(J_{0}^{\prime} C^{-1} J_{0}\right)^{-1} \geq\left(J_{0}^{\prime} C_{0}^{-1} J_{0}\right)^{-1}$ where $\geq$ stands for Löwner ordering) and the equality holds if and only if $D=0$, and this occurs when $C=C_{0}$, the true variance of $g_{i}$ when $E\left(g_{i}\right)=0$. The efficiency proof here is a standard result as in estimating function theory (Godambe, 1960) and generalized method of moments (Hansen, 1982). This argument can also explain why it is important to incorporate correlation for estimation instead of assuming independence structure.

\section{Proof of Theorem 3}

This proof is similar to the proof of Theorem 1 in Qu et al. (2000). 
BetaO

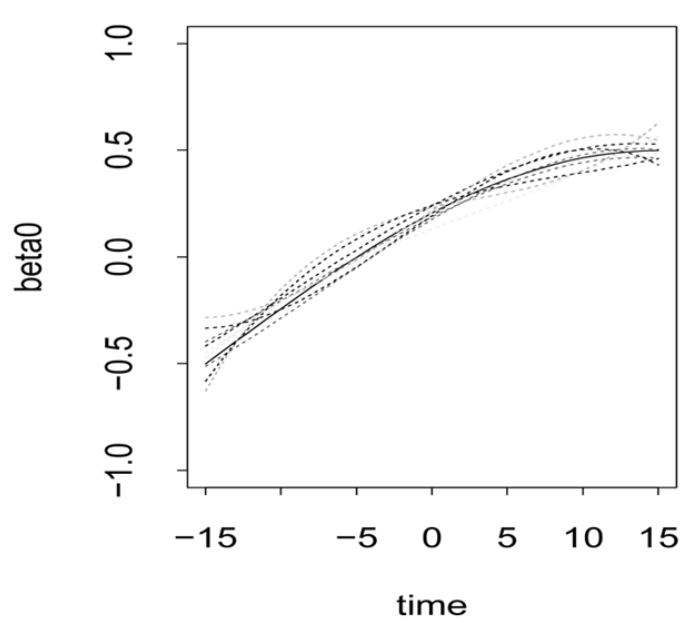

Beta2

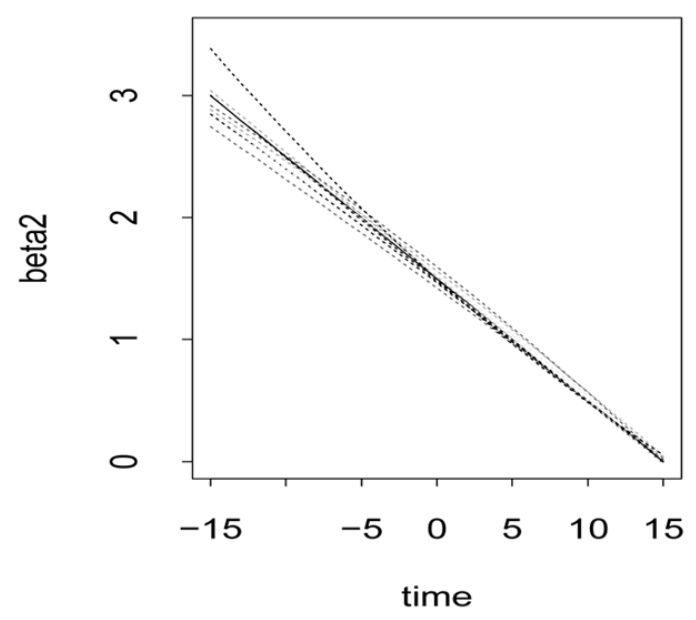

Beta1

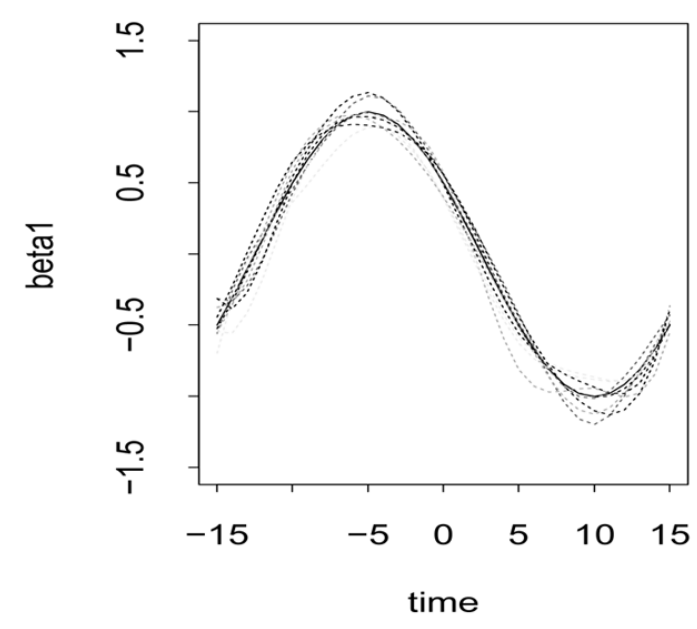

\section{Beta3}

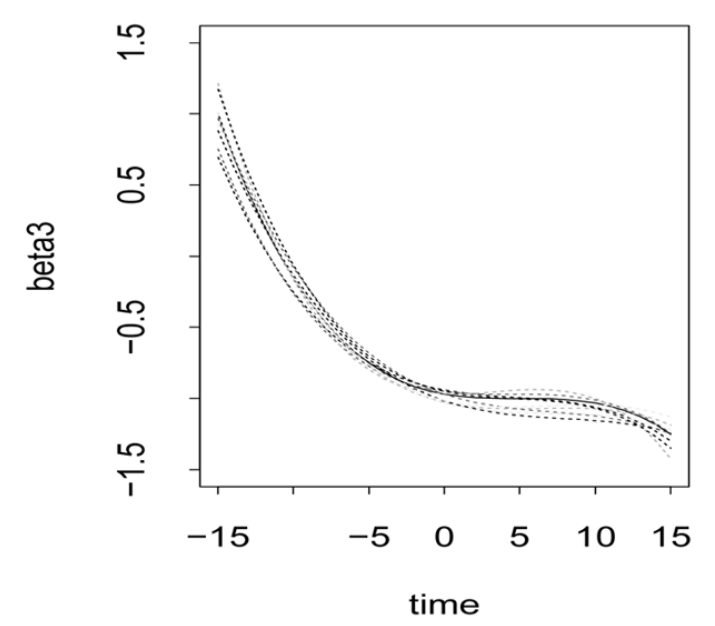

\section{Figure 1.}

For binary responses, fitted varying coefficient curves corresponding to 9 deciles of mean absolute deviation of errors, from 1000 simulations. The solid lines are true coefficient curves. 

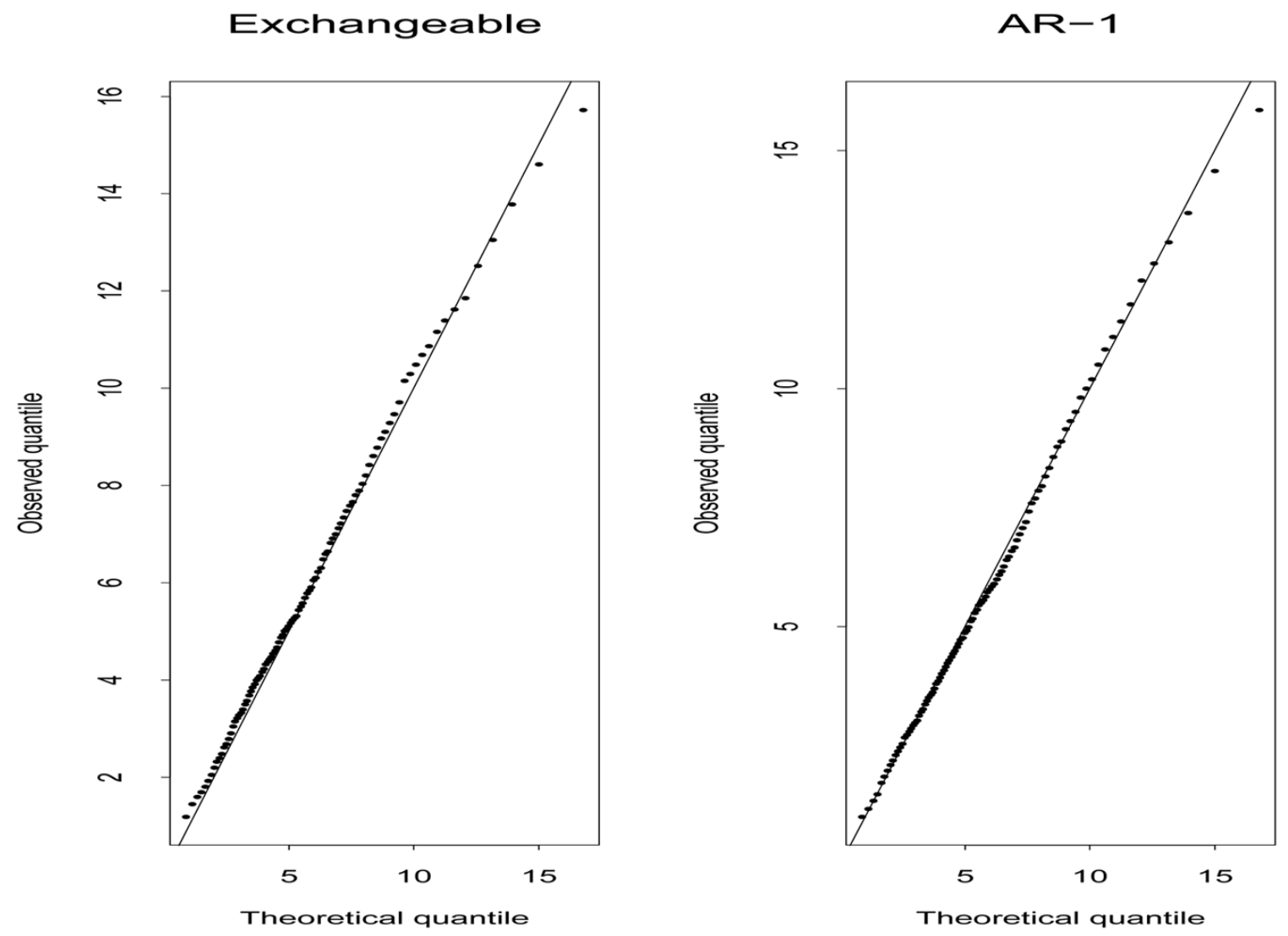

Figure 2.

For binary responses, quantile-quantile plots for test statistics $Q(\tilde{\beta})-Q(\hat{\beta})$ versus $\chi_{6}^{2}$ under $H_{0}$ : $\beta_{1}$ is constant over time, from 1000 simulations: (a) assume exchangeable working correlation; (b) assume AR-1 working correlation. 


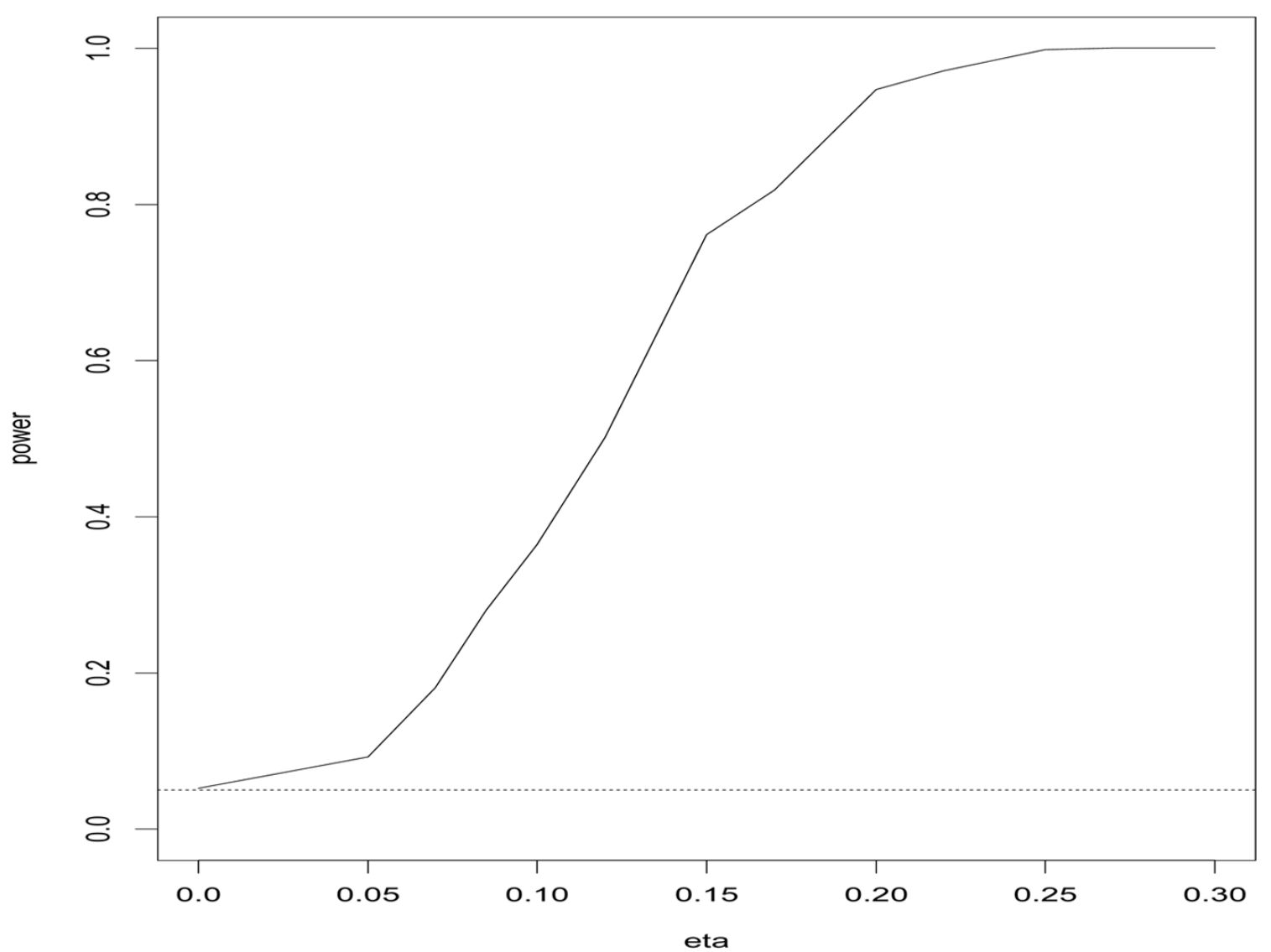

Figure 3.

For binary responses, power of quadratic inference function against $\eta$ for testing $H_{0}$ : $\beta_{1}$ is constant over time, from 1000 simulations. 
Beta0

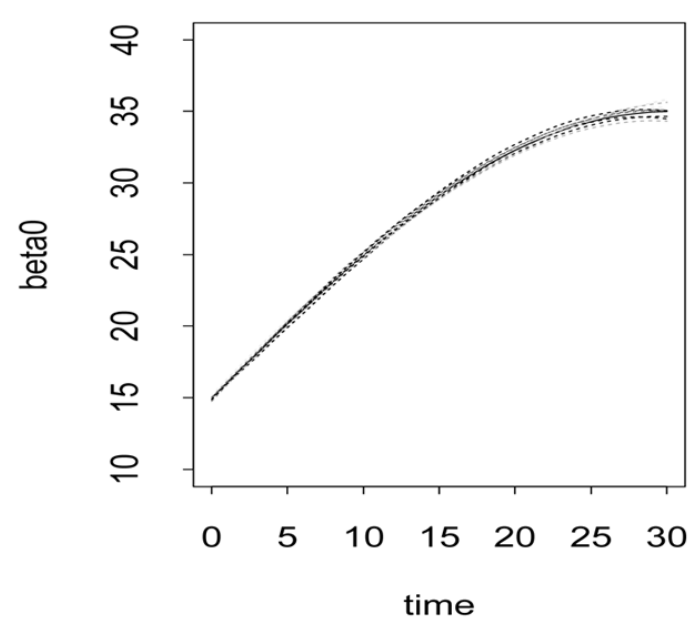

Beta2

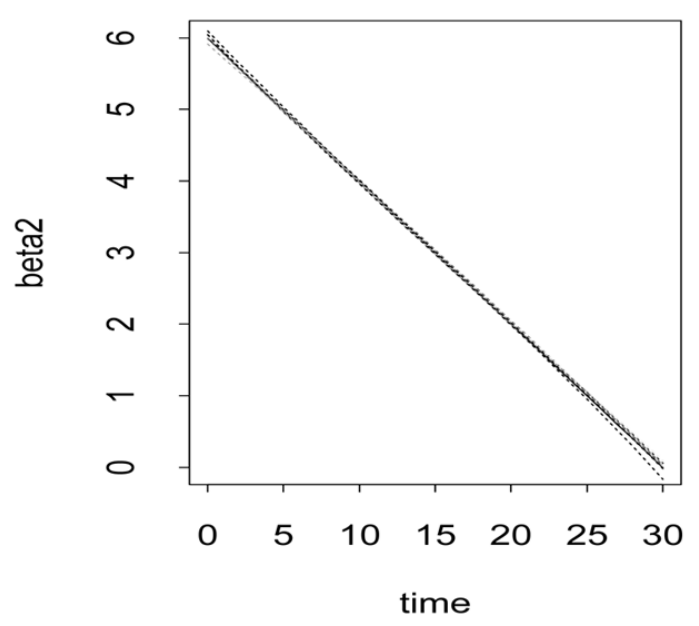

Beta1

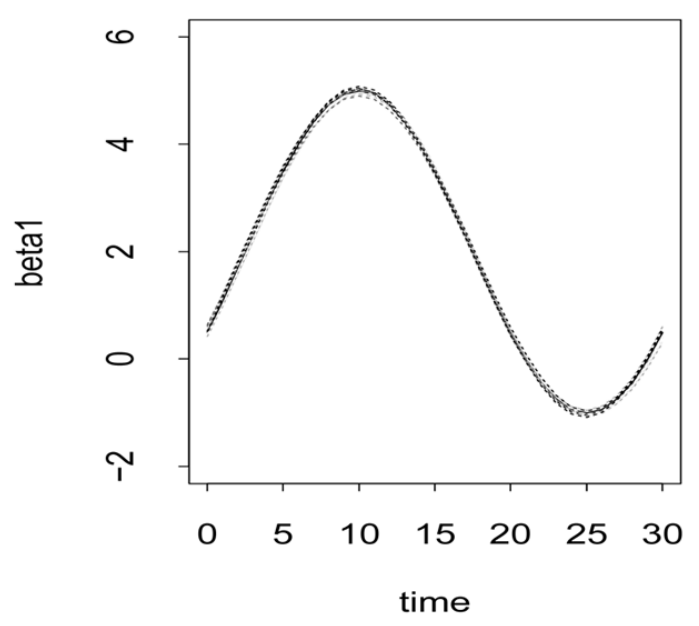

Beta3

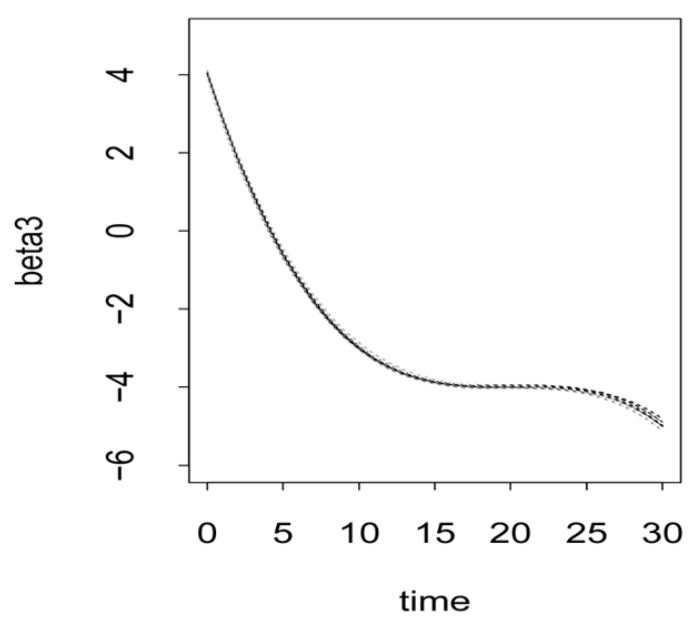

Figure 4.

For continuous responses, fitted varying coefficient curves corresponding to 9 deciles of mean absolute deviation of errors from 1000 simulations. The solid lines are true coefficient curves. 

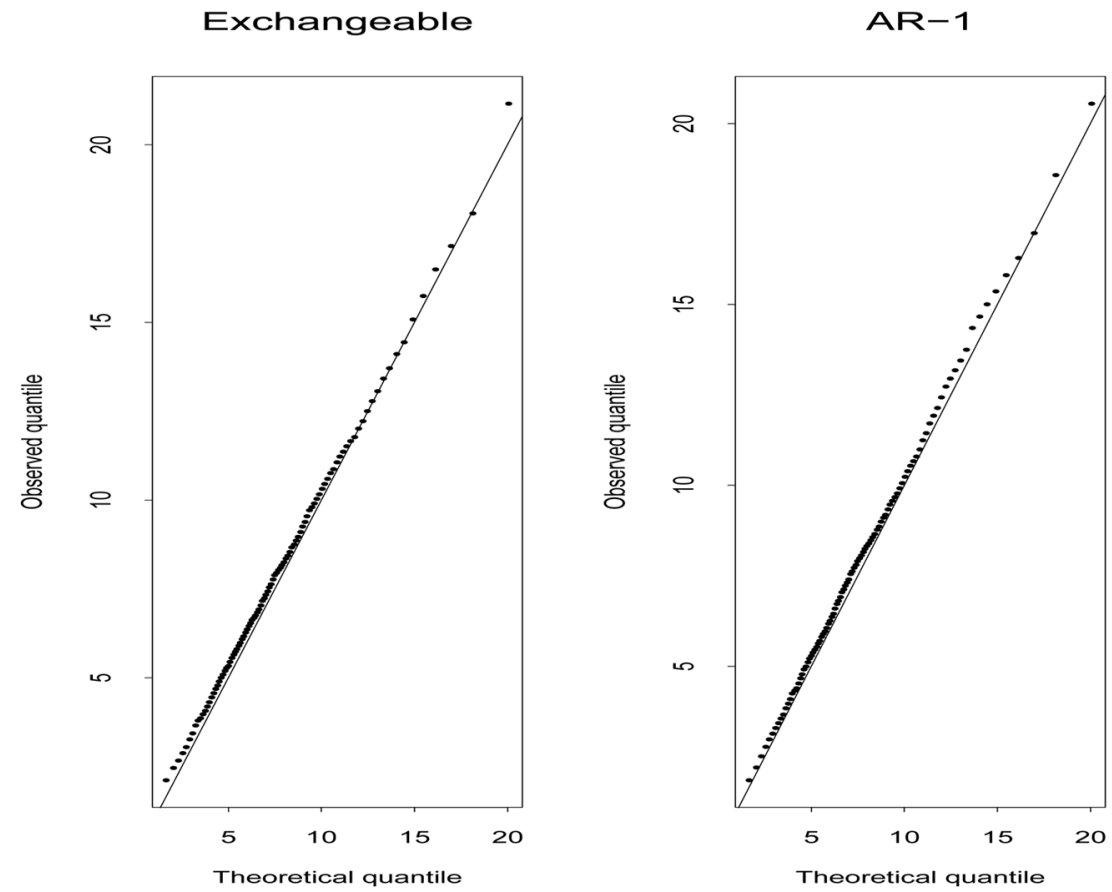

Figure 5.

For continuous responses, quantile-quantile plots for test statistics $Q(\tilde{\beta})-Q(\hat{\beta})$ versus $\chi_{8}^{2}$ under $H_{0}: \beta_{1}$ is constant over time from 1000 simulations: (a) assume exchangeable working correlation; (b) assume AR-1 working correlation. 
BetaO

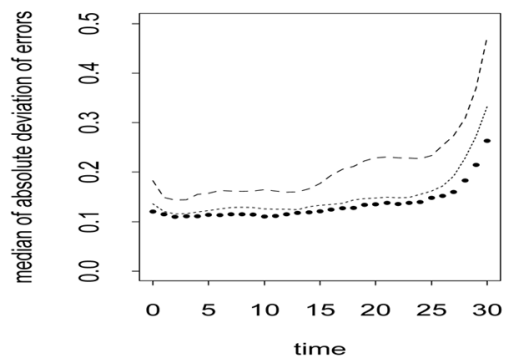

Beta2

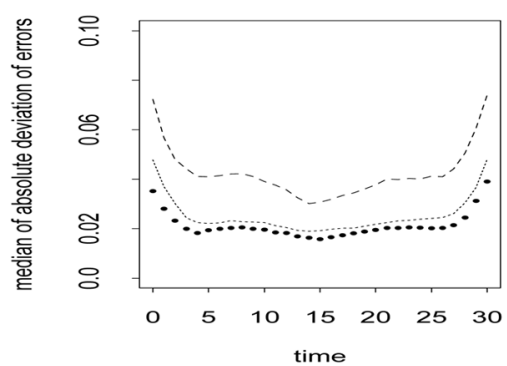

Beta1

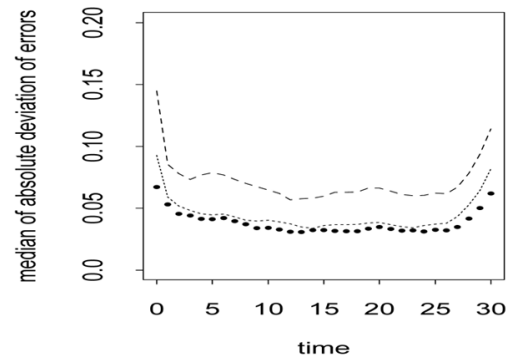

Beta3

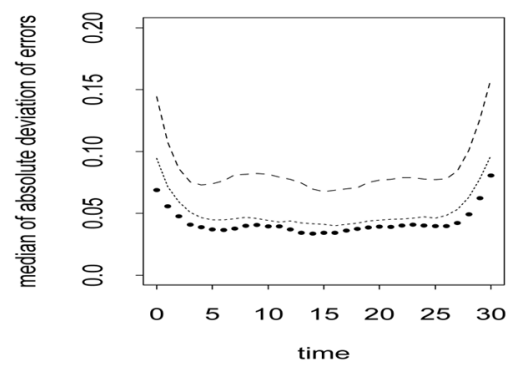

Figure 6.

For continuous responses, the median of absolute deviation of errors between fitted and true values from 1000 simulations for three different working correlation structures. The bold dotted line is from exchangeable correlation, the dotted line is from AR-1 correlation and the dash line is from independent structure 


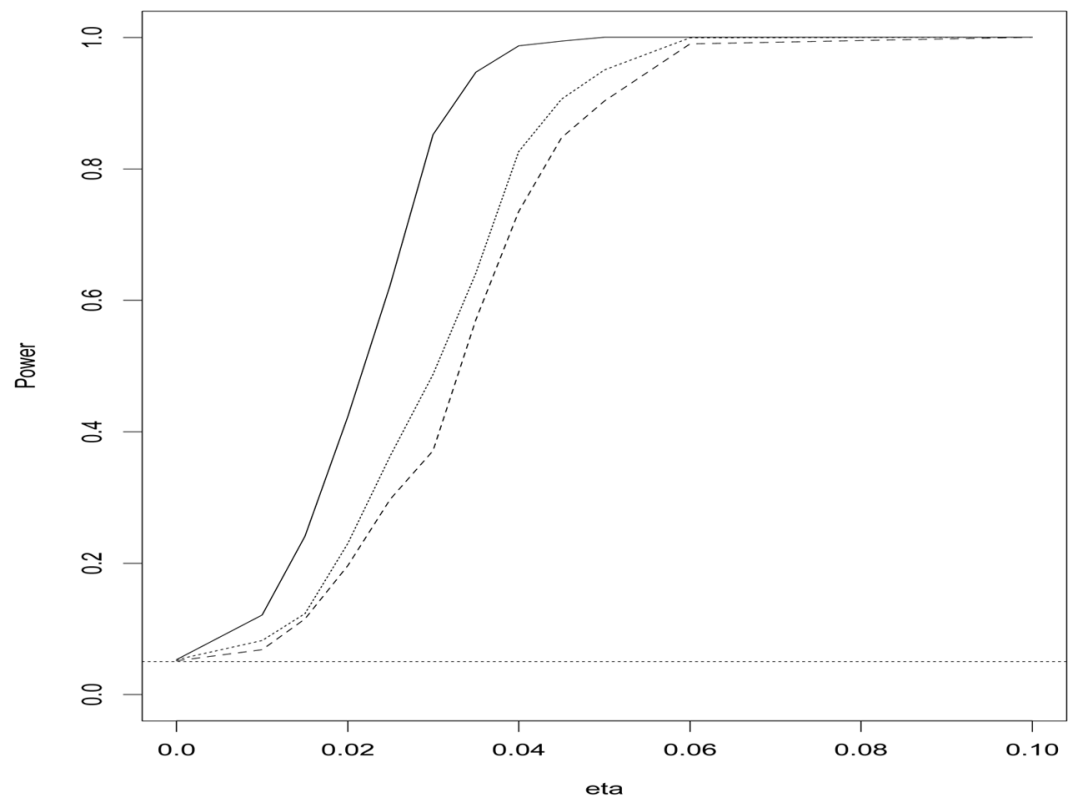

Figure 7.

For continuous responses, power of quadratic inference function against $\eta$ for testing $H_{0}: \beta_{1}$ is constant over time from 1000 simulations. The solid line is the power using exchangeable correlation, the dotted line is the power using AR-1 correlation and the dash line is the power using independent structure. The true correlation structure is exchangeable. 
Intercept effect

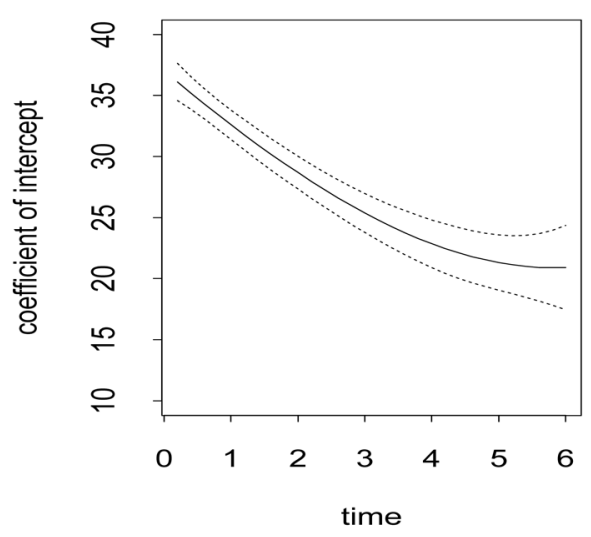

Age effect

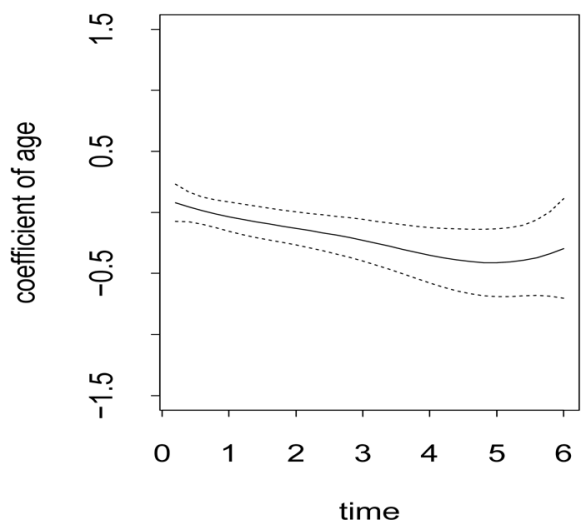

Smoking effect

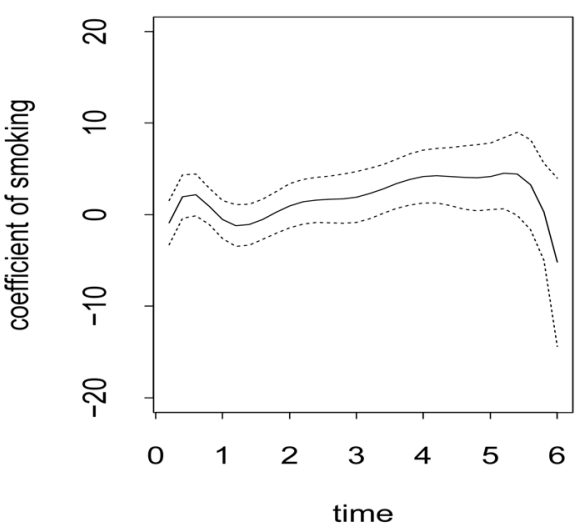

Pre-CD4 effect

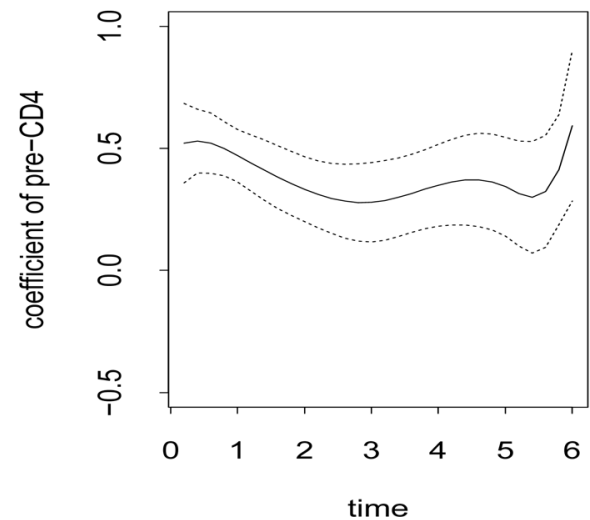

Figure 8.

Fitted varying coefficients for AIDs data, where solid lines are fitted curves and dotted lines are $95 \%$ pointwise confidence intervals. 
Table 1

Hypothesis testing for AIDs data, comparisons between Huang et al.'s (2002) bootstrap approach and the quadratic inference function approach

\begin{tabular}{lcccc}
\hline & $\begin{array}{c}\text { Bootstrap } \\
\text { (Independent) }\end{array}$ & Quadratic inference function (Exchangeable) \\
\hline Null hypothesis & $p$-value & T & d.f \\
Constant baseline & 0.000 & 81.9 & 3 & 9.000 \\
Smoking has no effect & 0.176 & 13.0 & 7.7 & 5 \\
Age has no effect & 0.301 & 12.9 & 0.163 \\
Constant Pre-CD4 & 0.059 & $0.045^{*}$ & 6 \\
\hline
\end{tabular}

$\mathrm{T}$ : test statistic defined in (12)

d.f.: degrees of freedom 